\title{
Erholung der deutschen Wirtschaft kommt 2022
}

Corona bestimmt die Wirtschaftsentwicklung seit knapp zwei Jahren. Ähnlich den Pandemie- bzw. Lockdown-Wellen ist seitdem auch der Konjunkturverlauf. Im internationalen Vergleich hat sich die deutsche Wirtschaft bislang nur relativ langsam von den pandemiebedingten Einbrüchen im Frühjahr 2020 und Anfang 2021 erholt. Nach dem 3. Quartal 2021 war das preis- und saisonbereinigte Bruttoinlandsprodukt (BIP) immer noch um gut $1 \%$ niedriger als im 4. Quartal 2019 vor Ausbruch der Pandemie. Andere Industrieländer, allen voran China und die USA, wie auch der Welthandel haben das Vorkrisenniveau bereits wieder überschritten; das hat zu den Materialengpässen und Logistikproblemen beigetragen. Und in den vergangenen Wochen hat sich trotz der erreichten Impffortschritte eine vierte Infektionswelle ausgebreitet, die die vorangegangenen gemessen an den Infektionszahlen noch weit übertrifft. Dies und vorgenannte Material- und Lieferprobleme bremsen den Aufholprozess erneut. Davon besonders betroffen sind vor allem die personenbasierten Dienstleistungen, insbesondere Handel und Gastronomie, sowie die Industrie, vor allem der Automobilbau. Im Schlussquartal 2021 dürfte die gesamtwirtschaftliche Leistung deshalb kaum zunehmen. Im Jahresdurchschnitt 2021 wird das Wachstum des realen BIP so lediglich $23 \% 4 \%$ betragen, nach einem Rückgang um 4,6\% im Jahr 2020.

2021 haben überdies die Inflationsrisiken merklich zugenommen. Besonders kräftig, teils zweistellig, sind die Preise auf den Vorstufen gestiegen. Aber auch die Anstiegsrate der Verbraucherpreise hat sich deutlich erhöht, zuletzt auf $5,2 \%$. Zunächst waren dafür Sonderfaktoren verantwortlich, wie die Wiederanhebung der im zweiten Halbjahr 2020 reduzierten Mehrwertsteuer, die Anfang 2021 neu eingeführte $\mathrm{CO}_{2}$-Abgabe sowie die im Vorjahrsvergleich stark erhöhten Energiepreise. Zur gleichen Vorjahreszeit waren Erzeuger- wie auch Verbraucherpreise gesunken. Aber auch krisenbedingte Angebotsengpässe auf manchen Rohstoff- und Warenmärkten haben 2021 zum Preisauftrieb beigetragen. 2022 werden die Sondereffekte nach und nach wegfallen und an den Märkten, an denen es zu Überhitzungen gekommen ist, kann es teils auch zu Preisrückgängen kommen. Bedeutsam für die Eindämmung der Inflationsrisiken ist auch, dass die Tarifpolitik hierzulande

(C) Der/die Autor:in 2021. Open Access: Dieser Artikel wird unter der Creative Commons Namensnennung 4.0 International Lizenz veröffentlicht (creativecommons.org/licenses/by/4.0/deed.de).

Open Access wird durch die ZBW - Leibniz-Informationszentrum Wirtschaft gefördert. die bislang temporären Preissteigerungen nicht zum Anlass für erhöhte Lohnforderungen genommen hat.

Seit Herbst hat sich zwar eine neue Pandemiewelle entwickelt, der Impfumfang der Bevölkerung wie auch politische Erklärungen lassen jedoch - bislang - erwarten, dass es in diesem Winter keinen erneuten pauschalen Lockdown geben wird. Allerdings sind Einschränkungen für bestimmte Personengruppen, insbesondere Ungeimpfte, und Großveranstaltungen denkbar. Den Aufholprozess vorläufig weiter beeinträchtigen dürften zudem die noch andauernden Lieferengpässe in der Industrie und der Bauwirtschaft, denn die Auftragslage dort ist gut. Die vierte Welle sowie die Material- und Lieferprobleme werden die Erholung noch bis ins Frühjahr 2022 dämpfen. Mit Nachlassen dieser Effekte sollte aber im Sommerhalbjahr 2022 der Erholungsprozess, verbunden mit Nachholeffekten, nochmal zusätzlichen Schwung bekommen.

Dafür dürfte nicht zuletzt die aufgestaute Konsum- und Investitionsnachfrage sorgen. Die privaten Haushalte haben ihre Kaufzurückhaltung während der vorangegangenen Lockdowns zu lockern begonnen und sie dürften ihr Konsumverhalten weiter normalisieren und ihre Sparquote reduzieren. Die Unternehmenserwartungen für die künftige Entwicklung sind ebenfalls recht positiv, sodass sich auch die Investitionsbereitschaft wieder erhöhen dürfte. Die Einstellungsbereitschaft der Unternehmen hat bereits zugenommen, die Zahl der sozialversicherungspflichtig Beschäftigten ist schon wieder über Vor-Corona-Niveau. Die öffentlichen Ausgaben werden schon deshalb kaum mehr steigen, weil die pandemiebedingten finanziellen Hilfen nach und nach auslaufen. Die neue Regierungskoalition hat zwar umfangreiche Investitionen angekündigt, deren Umsetzung benötigt aber einige Zeit und auf kommunaler Ebene wird wohl die pandemiebedingte Neuverschuldung die Investitionstätigkeit dämpfen. Die Weltwirtschaft entwickelt sich bereits dynamischer, sodass die Auslandsnachfrage und damit die Exporte deutlich zunehmen dürften. Im Zuge des Aufholprozesses hierzulande werden aber auch die Importe spürbar steigen, sodass die Wachstumsimpulse von außen insgesamt gering sein werden. Alles in allem ist mit einer Fortsetzung der Erholung der deutschen Wirtschaft 2022 zu rechnen. Die Dynamik des Aufholprozesses wird nach Erreichen des Vor-Corona-Niveaus allerdings allmählich nachlassen und Richtung Potenzialpfad zurückschwenken. Dies wird sich dann vor allem im Laufe des Jahres 2023 bemerkbar machen. Im Jahresdurchschnitt 2022 
Tabelle 1

\section{Eckdaten für Deutschland}

Veränderung in \% gegenüber dem Vorjahr

\begin{tabular}{|c|c|c|c|c|}
\hline & 2020 & 2021 & 2022 & 2023 \\
\hline Bruttoinlandsprodukt ${ }^{1}$ & $-4,6$ & 2,8 & 3,5 & 2,0 \\
\hline Private Konsumausgaben & $-5,9$ & 0,0 & 4,7 & 2,1 \\
\hline Staatliche Konsumausgaben & 3,5 & 3,0 & 1,0 & 1,0 \\
\hline Anlageinvestitionen & $-2,2$ & 1,6 & 3,9 & 4,2 \\
\hline Ausrüstungen & $-11,2$ & 3,6 & 4,3 & 6,4 \\
\hline Bauten & 2,5 & 1,6 & 2,7 & 2,8 \\
\hline Sonstige Anlagen & 1,0 & $-1,5$ & 6,6 & 4,1 \\
\hline Inlandsnachfrage & $-4,0$ & 2,5 & 3,2 & 1,9 \\
\hline Ausfuhr & $-9,3$ & 7,5 & 4,2 & 5,3 \\
\hline Einfuhr & $-8,6$ & 7,4 & 3,6 & 5,5 \\
\hline \multicolumn{5}{|l|}{ Arbeitsmarkt } \\
\hline Erwerbstätige & $-0,8$ & 0,0 & 0,7 & 0,5 \\
\hline Arbeitslose (in Mio.) & 2,70 & 2,61 & 2,33 & 2,23 \\
\hline Arbeitslosenquote $^{2}$ (in \%) & 5,7 & 5,5 & 4,9 & 4,7 \\
\hline Verbraucherpreise & 0,5 & 3,1 & 2,3 & 1,8 \\
\hline $\begin{array}{l}\text { Finanzierungssaldo des Staates } \\
\text { (in \% des BIP) }\end{array}$ & $-4,3$ & $-4,5$ & $-2,1$ & $-0,8$ \\
\hline Leistungsbilanzsaldo ${ }^{3}$ (in \% des BIP) & 7,0 & 6,8 & 6,9 & 7,2 \\
\hline
\end{tabular}

${ }^{1}$ Preisbereinigt. ${ }^{2}$ Arbeitslose in \% der inländischen Erwerbspersonen (Wohnortkonzept). ${ }^{3}$ In der Abgrenzung der Zahlungsbilanzstatistik.

Quellen: Statistisches Bundesamt; Deutsche Bundesbank; Bundesagentur für Arbeit; ab 2021 Prognose des HWWI.

wird das Wirtschaftswachstum etwa 31/2\% betragen, im Jahresdurchschnitt 2023 wird es sich auf $2 \%$ vermindern.

Die Beschäftigung wird bei dieser Entwicklung weiter zunehmen und die Zahl der Arbeitslosen merklich zurückgehen. Die Niveaus vor Pandemieausbruch dürften aber erst Anfang 2023 wieder erreicht werden. Das liegt nicht zuletzt daran, dass die Zahl der Selbstständigen, insbesondere der Kleinselbstständigen, seit Corona-Beginn um insgesamt rund $5 \%$ gesunken ist. Die Zahl der Angestellten ist, auch dank der erweiterten Kurzarbeiterregelungen, schon wieder etwa auf Vor-Corona-Niveau. Die Anstiegsrate der Verbraucherpreise wird 2022 wegen der dann wegfallenden Basiseffekte wieder niedriger ausfallen. Offen ist, wie schnell die in solch volatilen Zeiten stark schwankenden Rohstoff- und Materialpreise und auch Frachtraten sich stabilisieren bzw. sogar wieder sinken. Die Inflationsrate dürfte ab Jahresanfang 2022 zurückgehen und könnte im günstigen Fall nach der Jahresmitte 2022 sogar deutlich unterhalb von $2 \%$ fallen. Im Laufe von 2023 dürfte sie sich

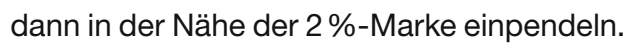

Die Corona-Pandemie wird die wirtschaftliche Entwicklung bis mindestens ins Jahr 2022 hinein weiter beeinflussen. Mit fortschreitenden Impfungen, umso mehr bei Einführung einer Impfpflicht, besteht die Chance, die Pandemie
Abbildung 1

Preisbereinigtes Bruttoinlandsprodukt

in Deutschland

Saison- und arbeitstäglich bereinigt

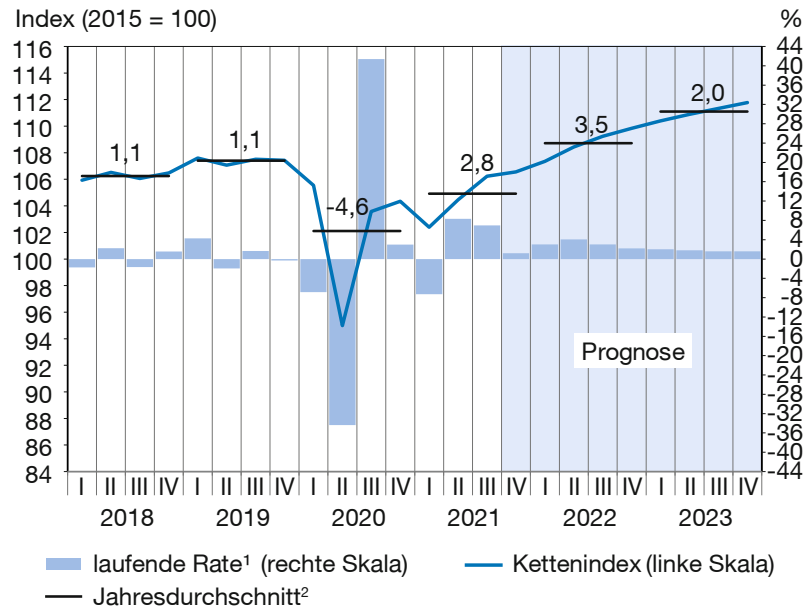

${ }^{1}$ Veränderung in \% gegenüber dem Vorquartal, auf Jahresrate hochgerechnet, rechte Skala. ${ }^{2}$ Zahlenangaben: Veränderung gegenüber dem Vorjahr in \%.

Quelle: Statistisches Bundesamt; ab 4.Quartal 2021 Prognose des HWWI.

in den Griff zu bekommen. Die Entwicklung mit der DeltaVariante hat aber auch gezeigt, dass die Auswirkungen von etwaigen neuen Mutanten - und mit Omikron ist bereits eine neue Mutante da - kaum abschätzbar sind. 2020 haben sich zudem durch die Materialknappheiten und Lieferkettenprobleme Inflationsrisiken aufgebaut. Sollten diese länger als erwartet andauern, bestünde die Gefahr von Kosten-Preis-Lohn-Spiralen und in der Folge geldpolitischer Restriktionen, welche die Erholung ausbremsen könnten; diese Gefahr besteht auch in anderen für die Weltwirtschaft wichtigen Industrieländern. Gewisse Unsicherheiten bringt auch der Regierungswechsel hierzulande mit sich. Die Koalitionsvereinbarungen schließen Steuererhöhungen aus und beinhalten die Einhaltung der Schuldenbremse wieder ab 2023. Insofern scheint finanzpolitische Stabilität gegeben, zumal mit der erwarteten Eindämmung der Pandemie ab voraussichtlich Sommer 2022 auch die fiskalischen Unterstützungszahlungen mehr und mehr auslaufen. Die Transformation hin zu mehr Klimaschutz und Digitalisierung beinhaltet sicherlich einiges Innovationsund Investitionspotenzial; deren Umfang wird aber auch von den Finanzierungsmöglichkeiten abhängen. Auch die Frage der Stabilität der Renten und deren Finanzierung ist - trotz der wohl geringeren Rentenerhöhungen 2022/23 noch nicht nachhaltig geklärt. Eine abrupte Erhöhung des Mindestlohns auf 12 Euro, d.h. um $25 \%$, könnte auch die Beschäftigung im Niedriglohnsektor dämpfen. 